PROCEEDINGS OF THE

AMERICAN MATHEMATICAL SOCIETY

Volume 128, Number 9 , Pages 2553-2559

S 0002-9939(00)05411-3

Article electronically published on February 29, 2000

\title{
A COUNTEREXAMPLE TO "AN EXTENSION OF THE VITALI-HAHN-SAKS THEOREM" AND A COMPACTNESS RESULT
}

\author{
GUY DEGLA
}

(Communicated by Christopher D. Sogge)

\begin{abstract}
We give a counterexample to "An extension of the Vitali-HahnSaks theorem" and from that highlight the sharp frame within which any attempt to change the version of such an extension should be possible. Lastly a sequential compactness criterion for Radon measures absolutely continuous with respect to a prescribed Radon measure defined on a locally compact separable metric space (taking into account the ideas of Hernandez-Lerma and Lasserre) is proved. The results deal with Radon measures but yield obvious corollaries on real (or vector-valued) Radon measures and so on functions with bounded variation on open subsets of $\mathbf{R}^{n}$.
\end{abstract}

\section{INTRODUCTION}

For the sake of clarity we start with some notations. Given a metric space $X$, $C(X)$ will denote the set of real-valued continuous functions on $X, C_{0}(X)$ the space (equipped with the sup-norm) of real-valued continuous functions on $X$ vanishing at infinity, $C_{c}(X)$ the subspace of $C_{0}(X)$ consisting of all continuous functions with compact support in $X, \mathcal{B}(X)$ the $\sigma$-algebra of Borel subsets of $X, \mathcal{M}^{+}(X)$ the set of Radon measures on $X$ (i.e., the set of Borel measures finite on every relatively compact Borel subset of $X$ ) and $\mathcal{M}_{b}^{+}(X)$ the set of bounded Radon measures on $X$. All the Borel measures will be assumed extended to their respective completions of $\mathcal{B}(X)$ but the outer measure of a Borel measure $\mu$ will be denoted by $\mu^{*}$.

Motivated by some version of the Vitali-Hahn-Saks theorem, the authors of 4, 5 stated recently (and somehow) the following proposition:

$[\oslash]$ Let $(X, \widetilde{\mathcal{B}}, \mu)$ be a $\sigma$-finite complete measure space, where $X$ is a locally compact separable metric space (in short a l.c.s.m space) and $\widetilde{\mathcal{B}}$ is the completion with respect to $\mu$ of the $\sigma$-algebra of Borel subsets of $X$. Let $\left(\lambda_{k}\right)_{k}$ and $\lambda$ be $\sigma$-finite (nonnegative) measures on $(X, \widetilde{\mathcal{B}})$ such that as $k \rightarrow \infty$

$$
\int_{X} v d \lambda_{k} \longrightarrow \int_{X} v d \lambda \forall v \in C_{0}(X) .
$$

Received by the editors October 1, 1998 .

2000 Mathematics Subject Classification. Primary 28A33; Secondary 28C15.

Key words and phrases. Radon, Borel, measures, absolute continuity, convergence, lower semicontinuity, differentiation, Lebesgue point, reflexivity. 
Assume also that every $\lambda_{k}$ is absolutely continuous with respect to $\mu$ (in short $\lambda_{k} \ll$ $\mu$ ) and

(ii)

$$
\lambda_{k}(B)=\int_{B} u_{k} d \mu, B \in \widetilde{\mathcal{B}} ; k=1, \ldots \text { with } \liminf _{k}\left\|u_{k}\right\|_{r}<\infty \text { and } r=\infty .
$$

Then $\lambda$ is absolutely continuous with respect to $\mu$.

Their idea is quite interesting (as shown by later developments), but the assumptions in $[\oslash]$ are not strong enough to guarantee always that $\lambda \ll \mu$ (as shown by Theorem 1 below). Moreover if (i) is satisfied, it should be understood that every element of $C_{0}(X)$ is $\lambda_{k}$ and $\lambda$ integrable so that the measures $\left(\lambda_{k}\right)_{k}$ and $\lambda$ must be finite (as shown by Theorem 2 below) and this prevents the application of $[\oslash]$ to unbounded $\sigma$-finite measures in contrast to a goal aimed in 4 4. Furthermore in order to take into account the aspect neglected in the proof of $[\oslash]$ appearing in 5] (while approximating the $\mu$-null set $B$ therein) one should also assume that $\mu \in \mathcal{M}^{+}(X)$ (as shown by Theorem 3 below) since that proof relies on the Lusin type criterion given in [3 IV.5] which requires that $X$ should be a countable union of open sets of finite $\mu$-measure. Hence another version of $[\oslash]$ is needed.

The aim of this paper is to revise and put their results in a general setting where $r \in] 1, \infty]$ with the observation that the case of $r=1$ is critical. Doing that, this paper also characterizes the finiteness of Borel measures by the integrability of functions vanishing at infinity on l.c.s.m. spaces, with the emphasis that this need not hold in an arbitrary l.c.m. space. Furthermore the proofs provided here are of independent interest and may be useful elsewhere.

\section{THEOREMS}

We start with the illuminating result which claims a counterexample to $[\oslash]$ and also shows somehow the significance of the work undertaken in [4], [5] and completed in this paper.

Theorem 1. There exists a $\sigma$-finite complete measure space $(X, \mathcal{B}(X), \lambda)$, where $X$ is a compact separable metric space, such that:

(1) for some closed subset $B$ of $X$ we have $\lambda(B)<\infty$ but $\lambda(G \backslash B)=\infty$ for every open subset $G$ of $X$ containing $B$.

(2) For some bounded Radon measures $\mu$ and $\mu_{k} ; k=1, \ldots$ on $X$ we have:

(i) $\mu_{k} \ll \lambda,\left\|\frac{d \mu_{k}}{d \lambda}\right\|_{\infty}=1 \forall k=1,2, \ldots$, and $\forall \varphi \in C_{0}(X), \int_{X} \varphi d \mu_{k} \rightarrow \int_{X} \varphi d \mu$ as $k \rightarrow \infty$.

(ii) $\mu$ is not absolutely continuous with respect to $\lambda$.

The following theorem prevents the application of $[\oslash]$ to unbounded measures in contrast to the main aim in 4 and also characterizes the finiteness of Borel measures on l.c.s.m. spaces.

Theorem 2. (1) Assume that $X$ is a locally compact separable metric space and $\mu$ a Borel measure on $X$. Then

$$
\mu(X)<\infty \Longleftrightarrow \int_{X} v d \mu<\infty, \forall v \in C_{0}(X) \text { with } v \geq 0 .
$$

(2) Nevertheless there exists a measure space $(X, \mathcal{B}(X), \mu)$, where $X$ is a locally compact (but not separable) metric space and $\mu$ a Borel measure such that: 
(i) $\mu(X)=\infty$.

(ii) $\int_{X} v d \mu<\infty, \forall v \in C_{0}(X)$ with $v \geq 0$.

Next we state a trivial fact but it allows to highlight the point neglected in [5].

Theorem 3. Let $X$ be a locally compact separable metric space and $\mu$ a Borel measure on $X$. Then $X$ is a union of a countable family of open sets with finite $\mu$-measure if and only if $\mu \in \mathcal{M}^{+}(X)$.

We separate the next theorem from its general version (see Theorem 6 below) because it deals with the Lebesgue measure (widely used in many applications) and its proof relies on some useful lemmas.

Theorem 4. Let $n$ be a positive integer, $\Omega$ a nonempty open set in $\mathbf{R}^{n}$ and $r \in$ ] $1, \infty]$. Denote the Lebesgue measure on $\Omega$ by $\ell^{n}$ and assume that $\left(\mu_{k}\right)_{k} \subset \mathcal{M}^{+}(\Omega)$ is a sequence of Radon measures such that: $\mu_{k} \ll \ell^{n}, \frac{d \mu_{k}}{d \ell^{n}} \in L^{r}\left(\Omega, \ell^{n}\right) \forall k$, and $c:=$ $\liminf _{k}\left\|\frac{d \mu_{k}}{d \ell^{n}}\right\|_{r}<\infty$. Then,

(1) there exists a subsequence $\left(\mu_{k_{j}}\right)_{j}$ of $\left(\mu_{k}\right)_{k}$ converging locally weakly to some $\mu \in \mathcal{M}^{+}(\Omega)$ (i.e., $\left.\int_{\Omega} \varphi d \mu_{k} \rightarrow \int_{\Omega} \varphi d \mu, \forall \varphi \in C_{c}(\Omega)\right)$ such that $\liminf _{j}\left\|\frac{d \mu_{k_{j}}}{d \ell^{n}}\right\|_{r}=$ c. Furthermore,

(2) $\mu \ll \ell^{n},\left\|\frac{d \mu}{d \ell^{n}}\right\|_{r} \leq \liminf _{j}\left\|\frac{d \mu_{k_{j}}}{d \ell^{n}}\right\|_{r}$ and $\int_{\Omega} \frac{d \mu}{d \ell^{n}}(x) d x \leq \liminf _{j} \int_{\Omega} \frac{d \mu_{k_{j}}}{d \ell^{n}}(x) d x$.

(3) If $r=\infty$, then also $\left.\int_{\Omega}\left|\frac{d \mu}{d \ell^{n}}(x)\right|^{p} d x \leq \liminf _{j} \int_{\Omega}\left|\frac{d \mu_{k_{j}}}{d \ell^{n}}(x)\right|^{p} d x, \forall p \in\right] 1,+\infty[$.

Observation 5. For $r=1$, there are many examples from probability theory and distribution theory which show that the above theorem cannot be extended.

Lastly we state a result which generalizes Theorem 4 and by all means takes into account the 'results' by O. Hernandez-Lerma and J. B. Lasserre.

Theorem 6. Let $X$ be a (nonempty) l.c.s.m. space, $\lambda \in \mathcal{M}^{+}(X)$ and $\left.\left.r \in\right] 1, \infty\right]$. Assume that $\mu_{k} \in \mathcal{M}^{+}(X) ; k=1, \ldots$ are such that: $\mu_{k} \ll \lambda, \frac{d \mu_{k}}{d \lambda} \in L^{r}(X, \lambda) \forall k$, and $c:=\liminf _{k}\left\|\frac{d \mu_{k}}{d \lambda}\right\|_{r}<\infty$. Then,

(1) there exists a subsequence $\left(\mu_{k_{j}}\right)_{j}$ of $\left(\mu_{k}\right)_{k}$ converging locally weakly to some $\mu \in \mathcal{M}^{+}(X)$ such that $\liminf _{j}\left\|\frac{d \mu_{k_{j}}}{d \lambda}\right\|_{r}=c<\infty$. Furthermore,

(2) $\mu \ll \lambda,\left\|\frac{d \mu}{d \lambda}\right\|_{r} \leq \liminf _{j}\left\|\frac{d \mu_{k_{j}}}{d \lambda}\right\|_{r}$ and

$$
\int_{X} \frac{d \mu}{d \lambda}(x) d \lambda(x) \leq \liminf _{j} \int_{X} \frac{d \mu_{k_{j}}}{d \lambda}(x) d \lambda(x) .
$$

(3) If $r=\infty$, then also

$$
\left.\int_{X}\left|\frac{d \mu}{d \lambda}(x)\right|^{p} d \lambda(x) \leq \liminf _{j} \int_{X}\left|\frac{d \mu_{k_{j}}}{d \lambda}(x)\right|^{p} d \lambda(x), \quad \forall p \in\right] 1,+\infty[.
$$

\section{Proofs}

Proof of Theorem 1. It is not hard to check that the following sets and measures satisfy the desired requirements: $X:=\{0\} \cup\left\{\frac{1}{j}: j=1,2, \ldots\right\}$ equipped with the usual metric inherited from the real line; $B:=\{0\} ; \lambda:=\sum_{j=1}^{\infty} \delta_{1 / j}$, where $\delta_{1 / j}$ is the Dirac measure concentrated on $\left\{\frac{1}{j}\right\}, \mu:=\delta_{0}$, and $\mu_{k}:=\delta_{1 / k}=1_{\{1 / k\}} \lambda$ for every positive integer $k$. 
Proof of Theorem 2. (1) We only show the sufficiency since the necessity is trivial. Suppose that $\int_{X} v d \mu<\infty, \forall v \in C_{0}(X)$ with $v \geq 0$. Then every element of $C_{0}(X)$ is $\mu$-integrable. Besides, since $X$ is a l.c.s.m. space, there exists a sequence of nonnegative continuous functions $0 \leq \varphi_{j} \leq 1$ with compact support converging pointwise to $1_{X}$ (and so does $\left(\varphi_{j}^{2}\right)_{j}$ ). For each $j$ define the linear functional $T_{j}: C_{0}(X) \rightarrow \mathbf{R}, f \mapsto \int_{X} \varphi_{j} f d \mu$. It is easy to see that $T_{j}$ is bounded and $\left\|T_{j}\right\| \leq \int_{X} \varphi_{j} d \mu$. Moreover for each $f \in C_{0}(X)$ we have $\left|T_{j}(f)\right| \leq \int_{X}|f| d \mu, \forall j$. Therefore by the Uniform Boundedness Principle we have $c:=\sup _{j}\left\|T_{j}\right\|<\infty$. Furthermore $\int_{X} \varphi_{j}^{2} d \mu=T_{j}\left(\varphi_{j}\right) \leq\left\|T_{j}\right\| \leq c$ so that by Fatou lemma we have $\mu(X)=\int_{X} \lim _{j} \varphi_{j}^{2} d \mu \leq \liminf _{j} \int_{X} \varphi_{j}^{2} d \mu \leq c<\infty$ completing the proof of the first part.

(2) Take $X=\mathbf{R}$ equipped with the discrete topology well-known as metrizable. Clearly, $X$ is a locally compact (but not separable) metric space and the set-function $\mu$ defined for each subset $B$ of $X$ by

$$
\mu(B)= \begin{cases}0 & \text { if } B \text { is at most countable } \\ \infty & \text { otherwise }\end{cases}
$$

satisfies trivially (i) $\mu(X)=\infty$ and (ii) $\int_{X} v d \mu=0, \forall v \in C_{0}(X)$ since one can check that the support of every element of $C_{0}(X)$ is at most countable (as only finite subsets of $X$ are compact). In fact

$C_{0}(X)=\left\{\sum_{l=-\infty}^{+\infty} a_{l} 1_{\left\{x_{l}\right\}}:\left(x_{l}\right)_{l} \subset X, x_{i} \neq x_{j}\right.$ for $\left.i \neq j,\left(a_{l}\right)_{l} \subset \mathbf{R}, \lim _{|l| \rightarrow \infty} a_{l}=0\right\}$.

Proof of Theorem 4. We first show the following lemma which characterizes the support of the singular part (relative to the Lebesgue measure) of a Radon measure defined on an open subset of $\mathbf{R}^{n}$.

Lemma. Let $n$ be a positive integer, $\Omega$ a nonempty open subset of $\mathbf{R}^{n}, \mu \in \mathcal{M}^{+}(\Omega)$ with the Lebesgue decomposition $\mu=\mu^{a}+\mu^{s}$ where $\mu^{a} \ll \ell^{n}$ and $\mu^{s} \perp \ell^{n}$ (i.e., $\mu^{s}$ is mutually singular with $\left.\ell^{n}\right)$. Set $S=\left\{x \in \Omega: \limsup _{\rho \rightarrow 0^{+}} \frac{\mu(B(x, \rho))}{\ell^{n}(B(x, \rho))}=\infty\right\}$ and define for each positive number $t$ the subsets $E_{t}=\left\{x \in \Omega: \limsup _{\rho \rightarrow 0^{+}} \frac{\mu(B(x, \rho))}{\ell^{n}(B(x, \rho))}>t\right\}$ and $F_{t}=\left\{x \in \Omega: \limsup _{\rho \rightarrow 0^{+}} \frac{\mu(B(x, \rho))}{\ell^{n}(B(x, \rho))}<t\right\}$. Then $\left(E_{t}, F_{t}, S\right.$ are Borel subsets and) we have:

(i) for every open subset $U$ of $\Omega, \ell^{n}\left(U \cap E_{t}\right) \leq \frac{1}{t} \mu\left(U \cap E_{t}\right)$ and $\mu\left(U \cap F_{t}\right) \leq$ $t \ell^{n}\left(U \cap F_{t}\right)$

(ii) $\ell^{n}(S)=0 \quad$ (so that $\mu^{a}$ is concentrated in $\Omega \backslash S$ ) and $\mu^{s}$ is concentrated in $S$.

Proof. It is a routine in measure theory to prove that such subsets are Borel.

(i) We omit this proof which simply relies on the classical application of the Vitali covering lemma (see, e.g., 7, p. 13])

(ii) We show that $\ell^{n}(S)=0$. Let $\left(\Omega_{j}\right)_{j \geq 1}$ be a sequence of open and relatively compact subsets of $\Omega$ such that $\bigcup_{j} \Omega_{j}=\Omega$. Therefore $\ell^{n}(S) \leq \sum_{j} \ell^{n}\left(S \cap \Omega_{j}\right)$ and thus it suffices to show that $\ell^{n}\left(S \cap \Omega_{j}\right)=0 \forall j$. Fix $j$ (arbitrarily) and let $\tau \in] 0, \infty\left[\right.$. Then by (i) we have $\ell^{n}\left(\Omega_{j} \cap E_{\tau}\right) \leq \frac{1}{\tau} \mu\left(\Omega_{j} \cap E_{\tau}\right)$ which implies that $\ell^{n}\left(\Omega_{j} \cap S\right) \leq \frac{1}{\tau} \mu\left(\Omega_{j}\right)$ since $S \subset E_{\tau}$. Letting $\tau \rightarrow \infty$ we get $\ell^{n}\left(S \cap \Omega_{j}\right)=0$ since $\mu\left(\Omega_{j}\right) \leq \mu\left(\bar{\Omega}_{j}\right)<\infty$ as $\bar{\Omega}_{j} \subset \Omega$ is compact and $\mu \in \mathcal{M}^{+}(\Omega)$. 
We show that $\mu^{s}$ is concentrated in $S$. It suffices to prove that $\mu^{*}(A)=0$ whenever $A \subset \Omega \backslash S$ and $\left(\ell^{n}\right)^{*}(A)=0$. Let $A \subset \Omega \backslash S$ be such that $\left(\ell^{n}\right)^{*}(A)=0$. Then $A$ is $\ell^{n}$-measurable and we can write $\ell^{n}(A)=0$. Moreover $A=\bigcup_{k=1}^{\infty}\left(A \cap F_{k}\right)$ and so it suffices to show that $\mu^{*}\left(A \cap F_{k}\right)=0 \forall k$. Now fix $k$ arbitrarily and set $A_{k}:=A \cap F_{k}$. Then $\ell^{n}\left(A_{k}\right)=0$ so that for every $\epsilon>0$ (fixed for a moment) there exists an open subset $U$ of $\Omega$ with $A_{k} \subset U$ and $\ell^{n}(U)<\epsilon$. This implies that $A_{k} \subset$ $F_{k} \cap U$ and by (i) we have $\mu^{*}\left(F_{k} \cap U\right) \leq k \ell^{n}\left(F_{k} \cap U\right)$ so that $\mu^{*}\left(A_{k}\right) \leq k \ell^{n}(U) \leq k \epsilon$. Therefore $\mu^{*}\left(A_{k}\right) \leq k \epsilon$ and letting (now) $\epsilon \rightarrow 0^{+}$we get $\mu^{*}\left(A_{k}\right)=0$ completing the proof of the lemma.

Now we are in a position to prove Theorem 4.

Proof of Theorem 4. Put for simplicity $L^{r}:=L^{r}\left(\Omega, \ell^{n}\right)$ and $u_{k}:=d \mu_{k} / d \ell^{n}$.

Case 1: Suppose $r \in] 1, \infty\left[\right.$. Then choose a subsequence of $\left(\mu_{k}\right)_{k}$ again denoted by $\left(\mu_{k}\right)$ such that $\lim _{k}\left\|u_{k}\right\|_{r}=c$. Therefore $\left(u_{k}\right)_{k}$ is bounded in the reflexive Banach space $L^{r}$ and it follows from the Eberlein-Smulyan theorem (see, e.g., 2, V.4.2; V.13.1]) that:

(1) there exists a subsequence $\left(\mu_{k_{j}}\right)_{j}$ of $\left(\mu_{k}\right)_{k}$ such that $\left(u_{k_{j}}\right)_{j}$ converges weakly to some $u \in L^{r}$ (i.e., $\int_{\Omega} v u_{k_{j}} d x \rightarrow \int_{\Omega} v u d x \forall v \in L^{r^{\prime}} ; 1 / r^{\prime}+1 / r=1$ ). It follows immediately that $u \geq 0 \ell^{n}$ a.e. in $\Omega$ (since $u_{k_{j}} \geq 0 \ell^{n}$ a.e. in $\Omega$ so that $\int_{A} u d x \geq$ $0 \forall A \in \mathcal{B}(\Omega)$ with $\bar{A} \subset \Omega$ compact $),\left(\mu_{k_{j}}\right)_{j}$ converges locally weakly ${ }^{\star}$ to $\mu=u \ell^{n} \in$ $\mathcal{M}^{+}(\Omega)$ and $\liminf { }_{j}\left\|u_{k_{j}}\right\|_{r}=c$. Furthermore,

(2) $\mu \ll \ell^{n}, d \mu / d \ell^{n}=u \in L^{r}$, by the lower semi-continuity of \|\|$_{r}$ with respect to the weak convergence in $L^{r}$ (see, e.g., [1 1, III.4]) we have $\|u\|_{r} \leq \liminf { }_{j}\left\|u_{k_{j}}\right\|_{r}$ and by the lower semi-continuity property of the local weak ${ }^{\star}$ convergence in $\mathcal{M}^{+}(\Omega)$ (see, e.g., [3, VIII.10.b] to be used appropriately) we have

$$
\int_{\Omega} u d x=\mu(\Omega) \leq \liminf _{j} \mu_{k_{j}}(\Omega)=\liminf _{j} \int_{\Omega} u_{k_{j}} d x
$$

(even if $r$ were $\infty$ ) completing the proof of this case.

Case 2: Suppose $r=\infty$. Then choose a subsequence of $\left(\mu_{k}\right)_{k}$ again denoted by $\left(\mu_{k}\right)$ such that $\lim _{k}\left\|u_{k}\right\|_{\infty}=c$. Therefore for each compact subset $K$ of $\Omega$ we have $\mu_{k}(K) \leq \ell^{n}(K) c, \forall k$. And since there exists a nondecreasing sequence of open and relatively compact subsets (of $\Omega$ ) whose union equals $\Omega$, the standard diagonal argument and the version of the Banach-Alaoglu theorem for Radon measures on compact sets (see, e.g., [3. VIII.5]) allow us to conclude that (1) there exists a subsequence $\left(\mu_{k_{j}}\right)_{j}$ of $\left(\mu_{k}\right)_{k}$ converging locally weakly ${ }^{\star}$ to some $\mu \in \mathcal{M}^{+}(\Omega)$. Observe at once that $\liminf _{j}\left\|u_{k_{j}}\right\|_{\infty}=c<\infty$.

(2) We now show that $\mu \ll \ell^{n}$. By the above lemma the singular part $\mu^{s}$ of $\mu$ in the Lebesgue decomposition relative to $\ell^{n}$ is concentrated in the subset $S=$ $\left\{x \in \Omega: \limsup _{\rho \rightarrow 0^{+}} \frac{\mu(B(x, \rho))}{\ell^{n}(B(x, \rho))}=\infty\right\}$; therefore it suffices to show that $S=\emptyset$. Indeed, let $x \in \Omega$ and take $\rho>0$ such that $\bar{B}(x, \rho) \subset \Omega$. Therefore by the lower semicontinuity property of the weak ${ }^{\star}$ convergence in $\mathcal{M}^{+}(\Omega)$ (see, e.g., [3, VIII.10.b]) we have $\mu(B(x, \rho)) \leq \liminf _{j} \mu_{k_{j}}(B(x, \rho))$. But $\mu_{k_{j}}(B(x, \rho)) \leq\left\|u_{k_{j}}\right\|_{\infty} \ell^{n}(B(x, \rho))$ (since $\mu_{k_{j}} \ll \ell^{n}$ ) and so $\frac{\mu(B(x, \rho))}{\ell^{n}(B(x, \rho))} \leq \liminf _{j}\left\|u_{k_{j}}\right\|_{\infty}$. Hence for each $x \in \Omega$ we have

$$
\limsup _{\rho \rightarrow 0^{+}} \frac{\mu(B(x, \rho))}{\ell^{n}(B(x, \rho))} \leq \liminf _{j}\left\|u_{k_{j}}\right\|_{\infty}<\infty
$$


which implies that $S=\emptyset$ so that $\mu \ll \ell^{n}$ and $u:=d \mu / d \ell^{n} \in L_{l o c}^{1}\left(\Omega, \ell^{n}\right)$. Moreover using Besicovitch's differentiation theorem (see, e.g., [7]) and (*) again we have $u \in L^{\infty}$ and $\|u\|_{\infty} \leq \liminf _{j}\left\|u_{k_{j}}\right\|_{\infty}$.

(3) Let $p \in] 1, \infty\left[\right.$. If $\liminf _{j} \int_{\Omega}\left|u_{k_{j}}\right|^{p} d x=\infty$, then the desired inequality is trivial.

Suppose that $\liminf \int_{\Omega}\left|u_{k_{j}}\right|^{p} d x<\infty$. Then it follows (anyway) from the proof of Case 1 that $\int_{\Omega}|u|^{p} d x \leq \liminf _{j} \int_{\Omega}\left|u_{k_{j}}\right|^{p} d x$ completing the proof.

Proof of Observation 5. We give an example which shows that Theorem 4 cannot be extended to the case of $r=1$. Let $\Theta$ be a nonnegative Borel (or simply continuous) function on $\mathbf{R}^{n}$ with compact support such that $\int_{\mathbf{R}^{n}} \Theta(x) d x=1$, define $u_{k}(x)=$ $k^{n} \Theta(k x) \geq 0, x \in \mathbf{R}^{n}$, for each positive integer $k$ and set $\mu_{k}=u_{k} \ell^{n}$. Then $\mu_{k} \ll$ $\ell^{n}, d \mu_{k} / d \ell^{n}=u_{k}$, and $\int_{\mathbf{R}^{n}} u_{k}(x) d x=1 \forall k$. But it is not hard (using a change of variables and the bounded convergence theorem) to see that $\lim _{k} \int_{\mathbf{R}^{n}} \varphi d \mu_{k}=$ $\varphi(0)=\int_{\mathbf{R}^{n}} \varphi d \delta_{0}$ for every bounded continuous function on $\mathbf{R}^{n}$ (i.e., $\left(\mu_{k}\right)_{k}$ converges narrowly to $\delta_{0}$ in $\left.\mathcal{M}_{b}^{+}\left(\mathbf{R}^{n}\right)\right)$ whereas $\delta_{0}$ is not absolutely continuous with respect to $\ell^{n}$.

Lastly we give the proof of Theorem 6 which does not show the subset in which the singular part is concentrated in general. The point is that the Vitali covering lemma relies on the geometry of $\mathbf{R}^{n}$; however we do not have an example of l.c.s.m. space, equipped with a Radon measure, for which the classical application of the Vitali covering lemma fails.

Proof of Theorem 6. For $r \in] 1, \infty[$ the proof is similar to the one of Theorem 4 .

Now suppose $r=\infty$. Then as in the proof of Theorem 4 we can conclude that

(1) there exists a subsequence $\left(\mu_{k_{j}}\right)_{j}$ of $\left(\mu_{k}\right)_{k}$ converging locally weakly ${ }^{\star}$ to some $\mu \in \mathcal{M}^{+}(X)$ such that $\liminf _{j}\left\|u_{k_{j}}\right\|_{\infty}=c<\infty$.

(2) We first show that $\mu \ll \lambda$. Since $X$ is a l.c.s.m. space, there exists a countable family of compact subsets $K_{l} ; l=1, \ldots$, of $X$ such that $K_{l} \subset \stackrel{\circ}{K}{ }_{l+1}, X=\bigcup_{l} \stackrel{\circ}{K}{ }_{l+1}$ and (since $\left.\lambda \in \mathcal{M}^{+}(X)\right) \lambda\left(K_{l}\right)<\infty, \forall l$. Now let $A$ be any subset of $X$ such that $\lambda^{*}(A)=0$. Then $A$ is $\lambda$-measurable and we can write $\lambda(A)=0$ so that for every $\epsilon>0$ (fixed for a moment) there exists an open subset $A_{\epsilon}$ of $X$ such that $A \subset A_{\epsilon}$ and $\lambda\left(A_{\epsilon}\right)<\epsilon$ (see, e.g., [3, V.5] or Lusin's criterion in [6, p.69]). It follows from the inequality $\mu_{k_{j}}\left(A_{\epsilon}\right) \leq\left\|u_{k_{j}}\right\|_{\infty} \lambda\left(A_{\epsilon}\right)$ and the lower semi-continuity property of the local weak* convergence in $\mathcal{M}^{+}(X)$ that $\mu\left(A_{\epsilon}\right) \leq \liminf _{j} \mu_{k_{j}}\left(A_{\epsilon}\right) \leq$ $\lambda\left(A_{\epsilon}\right) \liminf _{j}\left\|u_{k_{j}}\right\|_{\infty}$ which implies $\mu\left(A_{\epsilon}\right) \leq \epsilon c$ and so $\mu^{*}(A) \leq \epsilon c$. By letting (now) $\epsilon \rightarrow 0^{+}$we get $\mu^{*}(A)=0$ since $c<\infty$ by assumption. Thus $\mu \ll \lambda$ and $u:=$ $d \mu / d \lambda \in L_{\text {loc }}^{1}(X, \lambda)$. It follows from the Lebesgue-Radon-Nikodym differentiation theorem (see, e.g., [3, X.17]) and the countable covering of $X$ by $\left(K_{l}\right)_{l}$ that $\lambda$-almost every element of $X$ is a Lebesgue point for $\mu$ relative to $\lambda$, that is, for $\lambda$-almost every $x \in X, \lambda(B(x, \rho))>0, \forall \rho>0$ and $\lim _{\rho \rightarrow 0^{+}} \frac{\mu(B(x, \rho))}{\lambda(B(x, \rho))}=u(x) \in[0, \infty[$. Therefore $0 \leq u(x) \leq c=\liminf _{j}\left\|u_{k_{j}}\right\|_{\infty}$ for $\lambda$ almost every $x \in X$ (see, e.g., $\left.(*)\right)$. The rest follows as in the proof of Theorem 4 .

\section{ACKNOWLEDGEMENTS}

Many thanks are due to the staff (mostly to its Head) of the Sector of Functional Analysis and Applications of SISSA without whom this work would not have been done. The author should also like to thank his colleagues for their coorperations. 


\section{REFERENCES}

1. H. BREZIS : Analyse Functionnelle, Theorie et Applications, $2^{e}$ t., Masson, Paris 1983. MR 85a:46001

2. J.B. CONWAY : A Course in Functional Analysis, 2nd ed. Springer-Verlag New York, 1990. MR 91e:46001

3. J.L. DOOB : Measure Theory, Springer-Verlag New York, 1994. MR 95c:28001

4. O. HERNANDEZ-LERMA, J.B. LASSERRE : An Extension of the Vitali-Hahn-Saks Theorem, Proc. Amer. Math. Soc. 124(1996), 3673 - 3676. MR 97f:28012

5. O. HERNANDEZ-LERMA, J.B. LASSERRE : Correction to "An Extension of the VitaliHahn-Saks Theorem", Proc. Amer. Math. Soc. 126(1998), 949.

6. P. MALliAVIN, H. AIRAULT, L. KAY, G. LETAC : Integration and Probability, SpringerVerlag New York 1995. MR 97f:28001a

7. W.P. ZIEMER : Weakly Differentiable Functions, Springer-Verlag New York 1989. MR 91e:46046

Internatinal School for Advanced Studies (SISSA-ISAS), Via Beirut 2-4, 34014 TriESTE, ITALY

E-mail address: degla@sissa.it 